\title{
An Experimental Spectroscopic and Theoretic Study of Acetylbergenin
}

\author{
Gisele F. de Castro, Diego dos S. Freitas, Alberto S Marques \& YujiTakahata.
}

\section{Introduction}

The acetylbergenin was synthesized from bergenin extracted from the bark of Endopleura uchi, which extract is used by Amazonian community to treat infections ${ }^{1}$, and various biological activities have been proven, among them: anti-ulcerogenic, hepatoprotection, anti-HIV, obesity inhibitor, antioxidant, antimicrobial, etc $2,3,4,5,6$. However, bergenin is (a little) lipophilicity, and is poorly absorbed by the human gastrointestinal tract ${ }^{7}$. The study of acetylbergenin showed greater biological effect and greater efficacy in relation to bergenin $2,3,4,5$. This study expands the information on the molecule 3,4 , 8, 10, 11-penta-ortho-acetylbergenin through theoretical calculations and UV/Visible spectroscopy.

\section{Methods}

The sample of acetylbergenin was synthesized from the acetylation of bergenin as described in ${ }^{8}$. The molecular geometry optimization as crystallography 9, was performed using the Hartree - Fock method with base function $6-31 \mathrm{G}^{*}$, simulating the molecule in three different (environments settings): vacuum, ethanol and water. Subsequently, the excitation energies were calculated using the Time Dependent Density Functional Theory (TD-DFT) using functional B3LYP and base function 6-31G*. The sample[2] was subjected to crystallization procedure in methanol at $40{ }^{\circ} \mathrm{C}$ for purification. Spectra UV /Visible impure sample and sample recrystallized, (both in ethanol) at a concentration of $1 \times 10-4 \mathrm{M}$, were recorded on a spectrophotometer UV absorption/Visible New 2102 UV PC. In this way it intends to study the solvent effect mainly in the UV spectrum of the molecule, and analyze in detail the nature of the electronic transitions by comparing the theoretical and experimental spectra.

\section{Results and Discussion}

\section{UV ABSORPTION SPECTRUM}

\section{Vacuum simulation}

The theoretical spectrum of UV-Visible absorption acetilbergenin in vacuum (Figure 1.a) was obtained from the data of the DFT B3LYP calculation DT / 6-31G*. It was observed that it had, three bands $\mathrm{A}, \mathrm{B}, \mathrm{C}$ in the simulated spectra in vacuum. The energies $(\mathrm{nm})$ and f-values of peaks of the three bands are: $\mathrm{A}(257 \mathrm{~nm}, \mathrm{~F}$ $=0.0159), \mathrm{B}(238 \mathrm{~nm} \mathrm{f}=0.11689)$, and $\mathrm{C}(223 \mathrm{~nm} \mathrm{f}=$ $0.0488)$, respectively .

The band $\mathrm{B}$ is the most intense and the band $\mathrm{A}$ is of lower intensity. The band $\mathrm{B}$ consists of two transitions, $S_{1}$ and $S_{2}$ of low intensities. $S_{1}$ transition is due to transition D (138) (HOMO-3, n carbonyls, $\rightarrow \mathrm{V}(1)$ (LUMO , antibonding $\left(\pi^{*}\right)$, located on the aromatic) 
where $\mathrm{D}(138)$ is random orbital-138 doubly occupied, and V (1) is first virtual orbit. S2 is due to transition $\mathrm{D}(140)$ (HOMO-1 (orbital $(\pi)$ located on the aromatic ring)) to the LUMO+1 (antibonding $\left(\pi^{*}\right)$ of the aromatic ring). The band $\mathrm{B}$ is composed of two transitions, $\mathrm{S}_{3}$ and $\mathrm{S}_{4}$, high intensities. $\mathrm{S}_{3}$ transition occurs from the HOMO-2 orbital, (ring D-glucopyranose, n) to the orbital V (1 ), LUMO. The transition $\mathrm{S}_{4}$ of the HOMO orbital ( $\pi$ located on the aromatic ring) to the LUMO. The $\mathrm{C}$ band is composed of two transitions, $\mathrm{S}_{5}$ and $\mathrm{S}_{6}$ average intensities. $S_{5}$ transition is due to a transition part of the HOMO-6 orbital ( $n$, located on the aromatic ring) to the LUMO.

The S6 transition is due a transition orbital D (134) HOMO-7 (n, with major contribution in orbital $\mathrm{n}$ located in the $\delta$-lactone ring $\mathrm{V}(1)$ (LUMO).

\section{Ethanol simulation}

The UV-Vis spectrum experimental compound in ethanol solution 10-4M presented three bands with peaks at 219nm (B'), 259nm (A') and 309nm (X') (Figure 1d) and the experimental spectrum of recrystallized acetilbergenin presented four bands with maxima at $205 \mathrm{~nm}$ (C'), $225 \mathrm{~nm}$ (B'), 255nm (A') and 304nm (X') (Figure 1.e).
The two experimental spectra are similar in bands B' and A'. However, there are some differences between the two spectra. First, the presence of the small band $\mathrm{C}$ ', which is on the left shoulder of the intense band B'. In Figure 1.e spectrum as the spectrum in Figure 1.d, both do not have a band C'. It is very likely that the band C' did not appear in the compound spectrum before recrystallization (Figure 1.d), because of impurity. Second, the presence of the band $X^{\prime}$ is sharp in Figure 1.d, whereas in Figure 1.e, the band $X^{\prime}$ is not well defined. In the spectrum of the compound crystallized in Figure 1.e, the band $X^{\prime}$, or does not exist or the intensity of the band is so weak that it is hidden in the right side of the band. It is very likely that the band X' in the sample spectrum before recrystallization (Figure 1.d), is due to impurity. In the compound crystallized, the amount of impurity has been reduced. Therefore, the spectrum of the recrystallized compound (Figure 1.e), the intensity of $\mathrm{X}$ ' band significantly decreased and was almost invisible.

The theoretical spectrum of UV-Vis absorption acetilbergenin based on the result of the calculation by the B3LYP DFT DT / 6-31G * in ethanol for three bands, $\mathrm{A}, \mathrm{B}, \mathrm{C}$ (Figure 1.b) where the band $\mathrm{B}$ is the more intense, and then the band $A$ and band $C$ have lower intensity. The

\section{Espectros Teóricos; a),b),c)}

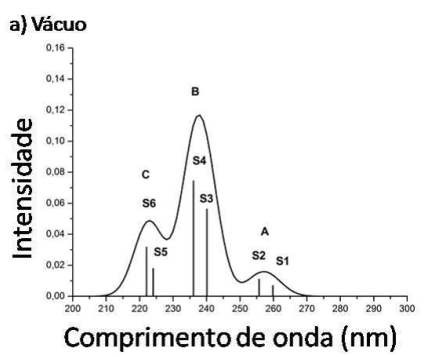

b) Etanol

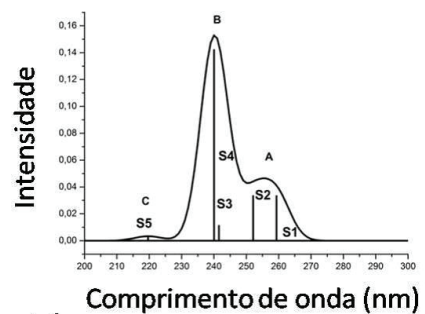

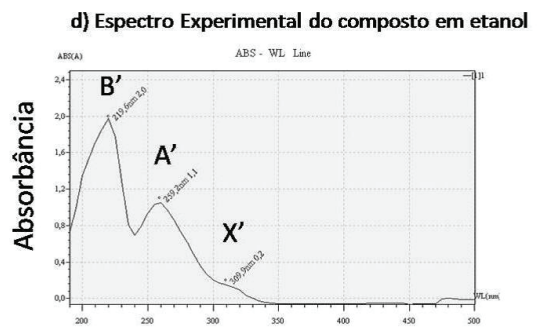

Comprimento de onda ( $\mathrm{nm}$ )
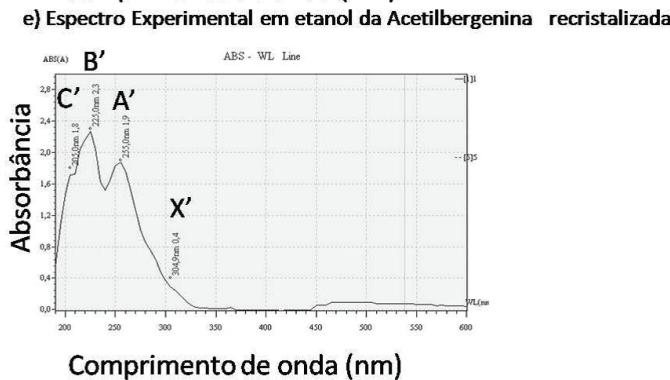

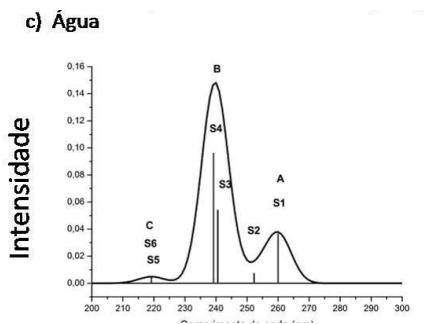

Comprimento de onda $(\mathrm{nm})$

Figure 1. Theoretical Absorption Spectrum; a) vacuum,b) ethanol, c) water, d) UV-Visible Eexperimental Absorption Spectrum, all in ethanol concentration $10^{-4} \mathrm{M}$ before crystallize, e)and rerecrystallized. 
energies (nm) and f-values of peaks are the three bands A $(255 \mathrm{~nm} \mathrm{f}=0.0464), \mathrm{B}(240 \mathrm{~nm} \mathrm{f}=0.152947)$ and C $(220$ $\mathrm{f}=0.0033$ ) respectively.

The experimental absorption spectrum of the UVVIS acetilbergenin in ethanol (Figure 1e) is similar to the theoretical spectrum (Figure 1.b). The bands A, B, $\mathrm{C}$ of the theoretical spectrum corresponding to bands A ', B', C ' respectively of the experimental spectrum. In the theoretical spectrum (Figure 1.b) there is a band on the right side of the band corresponding to the band $X^{\prime}$ of the experimental spectrum. We conclude that the band $X^{\prime}$ in the experimental spectrum in Figure 1.d and Figure 1.e is probably due to impurity. The band A of the theoretical spectrum consists of two transitions, S1 and $\mathrm{S} 2$ of medium intensity. The $\mathrm{S}_{1}$ transition is due to $\mathrm{D}(140)$ (HOMO-1, orbital $\pi) \rightarrow \mathrm{V}(1)$ (LUMO, antibonding orbital $\left(\pi^{*}\right)$ of aromatic ring). The $\mathrm{S}_{2}$ transition is due to a transition from $\mathrm{D}$ (139) (HOMO-2, n located on orbital D- glicopiranose ring) to the orbital V (1) (LUMO, $\pi *$ ).

The band B is composed of two transitions, $\mathrm{S}_{3}$ and $\mathrm{S} 4$, high intensities. $\mathrm{S}_{3}$ transition occurs a transition orbital D (135) (HOMO-6, n acetyl group of the aromatic ring) to the orbital V (1) (LUMO). The S4 transition is the transition part of the orbital D (141) (HOMO, $\pi$ located on the aromatic ring) to the orbital V (1) (LUMO).

The $\mathrm{C}$ band is composed of a transition, S5, low intensity. S5 transition is due to a transition of the orbital D (139) (HOMO-2, n ) located on the aromatic for the orbital $\mathrm{V}(2)\left(\mathrm{LUMO}+1\right.$, antibonding $\left.\pi^{*}\right)$ located on the aromatic ring. The photoactive region of the molecule are located on the aromatic ring region, where transitions occur between the HOMO, LUMO, HOMO-2 and LUMO+1 .Comparing the two theoretical spectra, one in vacuum (Figure 1.a) and another ethanol (Figure 1.b), it is observed that the relative intensity of the $\mathrm{C}$ band from the band B in Figure 1.b is smaller than the same in Figure 1.a.

\section{Water simulation}

The theoretical spectrum of the UV-VIS absorption acetilbergenin result of the calculation by DFT B3LYP / $6-31 \mathrm{G} *$ in water is shown in Figure 1c. There are three bands, $\mathrm{A}, \mathrm{B}$, and $\mathrm{C}$, in a theoretical spectrum, where the peak positions are the wavelengths at $260 \mathrm{~nm}$ ( $\mathrm{f}=$ $0,03791, \mathrm{~A}), 240 \mathrm{~nm}(\mathrm{f}=0.1480, \mathrm{~B})$ and $219 \mathrm{~nm}(\mathrm{f}=$ $0.0048, C)$. The A band consists of the transitions, $S_{1}$ and
$\mathrm{S}_{2}$ medium intensity. S1 V (1) (LUMO, $* \pi$ ) transition is due to transition $\mathrm{D}(140)$ (HOMO-1, $\pi$ ) where the orbital are located on the aromatic ring and $\mathrm{S} 2$ refers to transition $\mathrm{D}(139)$ (HOMO-2, n) $\square \mathrm{V}(1)$ (LUMO, $\pi^{*}$ ) leaving the carbonyl of D-glucopyranose ring. The band $\mathrm{B}$ is composed of two transitions, S3 and S4, high intensities. $\mathrm{S}_{3}$ transition occurs a transition orbital $\mathrm{D}(134)(\mathrm{HOMO}-$ $7, n$ ) located on the acetyl group of the aromatic ring to the orbital V (1) (LUMO, $\pi *$ ). The $\mathrm{S}_{4}$ transition is the transition part of the orbital D (141) (HOMO, $\pi$ ) for the orbital V (1) LUMO. The C band is composed of two transitions, $\mathrm{S}_{5}$, and $\mathrm{S}_{6}$, of low intensity. $\mathrm{S} 5$ transition is due to a transition of the orbital $\mathrm{D}(140)(\mathrm{HOMO}-1, \pi)$ to the orbital V(2) (LUMO $\left.+1, \pi^{*}\right)$. The transition S6 respect to transition $\mathrm{D}(134)(\mathrm{HOMO}-7) \rightarrow \mathrm{V}(1)$ LUMO.

\section{Solvent effect}

The theoretical analysis of the absorption spectra, in Figure 1, shows that comparatively the bands of simulation in ethanol (Figure 1.b) shifted to longer wavelengths relative to the vacuum spectrum (Figure 1.a), and in the water spectrum (Figure 1.c) it was reversed. This demonstrates that the nature of electronic transitions in bands $\mathrm{A}$ and $\mathrm{B}$ is predominantly of type $\pi \rightarrow \pi *$ in vacuum and in water, while band $C$ in the ethanol simulates the transition $\mathrm{n} \rightarrow \pi *$ predominates.

By visualizing the orbital calculated by DFT for acetilbergenin in vacuum, it was observed that the photoactive region of the molecule is located on the aromatic ring, where transitions occur between the HOMO $\rightarrow$ LUMO and HOMO-1 $\rightarrow$ LUMO +1 (Figure 2).
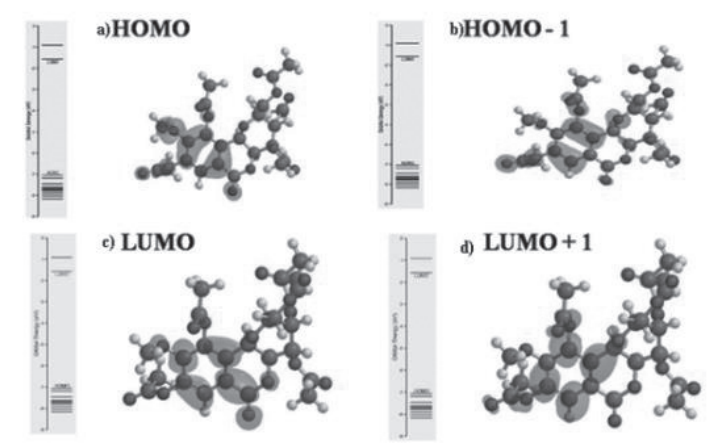

Figure 2. Molecular orbitals of acetilbergenin in vacuum. a)HOMO b) HOMO-1 c) LUMO d) LUMO+1 
The solvent change slightly altered the electrostatic potential map both in water (Figure 3.a) and ethanol (Figure 3.b). Probably this change was due to changes in the conformation of the molecule between these the dihedral were highlighted: $\mathrm{C}_{24} \mathrm{O}_{1} \mathrm{C}_{1} \mathrm{C}_{5}$ (water $=-43,97^{\circ}$ ; ethanol $=-43,45^{\circ} ;$ vacuum $\left.=-45,64^{\circ}\right), \quad \mathrm{C}_{15} \mathrm{O}_{10} \mathrm{C}_{16} \mathrm{C}_{17}$ (water $=\quad 9,12^{\circ}$; ethanol $=7,43^{\circ} ;$ vacuum $=12,62^{\circ}$ ), $\mathrm{C}_{13} \mathrm{C}_{12} \mathrm{O}_{8} \mathrm{C}_{11}$ (water $=-15,90^{\circ}$; ethanol $=-15,14^{\circ}$; vacuum = $-17,92^{\circ}$ ) and the dihedral $\mathrm{C} 20 \mathrm{O} 13 \mathrm{C} 21 \mathrm{O} 14$ (water $=-0,33^{\circ}$ ; ethanol $=2,39^{\circ}$; vacuum $=-1,84^{\circ}$ ) of the acetyl group of the aromatic ring where there was a change of plan in the middle ethanol.

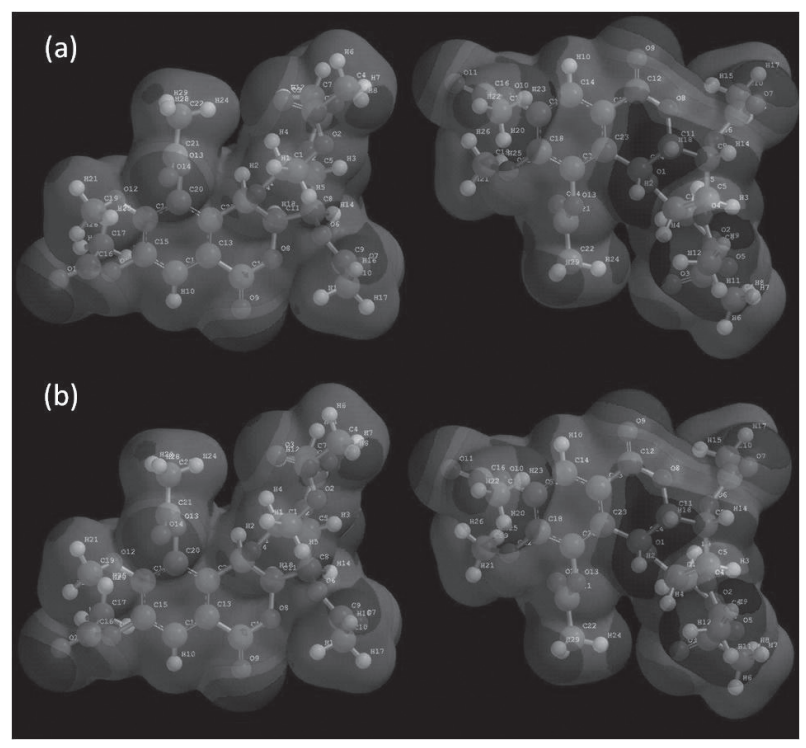

Figure 3: Map of the electrostatic potential of acetilbergenin molecule on a surface of $0.002 \mathrm{eV} / \mathrm{ua}^{3}$. a)water b) ethanol.

The dipole moment of acetilbergenin in water 7,8826 Debye (26,2931. $10^{-30}$ C.m) was larger than 7,57 Debye $\left(25,2331.10^{-30}\right.$ C.m $)$ in ethanol and in vacuum 6,07 Debye. The increase dipole highlights the transitions $n \rightarrow \pi^{*}$ with charge transfer character and also the molecular deactivation process in the excited electronic state to another in the ground state via the Forster mechanism ${ }^{10}$.

A comparison was made of the HOMO and LUMO energies in the three simulations and it was found that the HOMO energy that has suffered changes with respect to the vacuum simulation was ethanol and the LUMO energy $(-1.64 \mathrm{v})$ in water. Amid ethanol energy difference between HOMO and LUMO showed lower value indicating higher reactivity ${ }^{11}$. The change in polarizability, with respect to the vacuum simulation was small in ethanol of 0.07 and 0.08 in water (79.58) (Table 1).

Table 1. Some properties calculated DFT 6-31G*

\begin{tabular}{|c|c|c|c|}
\hline Acetilbergenin & Vacuum & Ethanol & Water \\
\hline Log P & $-0,76$ & $-0,76$ & $-0,76$ \\
\hline Polarizability & 79,50 & 79,57 & 79,58 \\
\hline $\begin{array}{c}\text { Dipole moment } \\
(\mathrm{D})\end{array}$ & 6,07 & 7,57 & 7,88 \\
\hline E-HOMO (eV) & $-7,06$ & $-6,95$ & $-7,04$ \\
\hline E-LUMO (eV) & $-1,58$ & $-1,56$ & $-1,64$ \\
\hline LUMO-HOMO & 5,48 & 5,39 & 5,4 \\
\hline
\end{tabular}

\section{Conclusions}

The results of the theoretical calculations showed consistency with the experimental data: (1) all three spectra have three bands, A, B, and C; (2) appearance and relative intensity of bands $\mathrm{A}$ and $\mathrm{B}$ are similar in the three spectra; (3) the relative intensity of band $\mathrm{C}$ in ethanol and in water is very weak compared to $1 \mathrm{a}$.The orbitals HOMO and LUMO are localized at the aromatic ring acetylbergenin, this being its most photoactive region.

The calculation showed that ethanol has changed to transition a long distance charge transfer, since in vacuum stemmed orbital (n) of $\delta$-lactone ring to the antibonding orbital $\left(\pi^{*}\right)$ of the aromatic ring and the orbiting ethanol was (n) the ring D - glucopyranose to the aromatic ring. This type of transition is usually the driving force responsible for the interaction of the molecule with biological material, because of this molecule is shown in more photoactive hydrophilic medium (ethanol, $\mathrm{f}=0.0333$ ) with oscillator strength greater than simulation vacuum (isolated molecule without interaction, $\mathrm{f}=0.0068$ ).

\section{Acknowledgments}

The authors are grateful for the support given from the CNPQ and MURAKI Foundation. 


\section{References}

1. CORRÊA, M. P. ; Dictionary of useful plants in Brazil and cultivated exotic in Rio de Janeiro, National Press, V.6; 1984.

2. BORGES, J. C. Collection and evaluation of antinociceptive and anti-inflammatory activities. Masters dissertation Federal University of Pará. 2010.

3. JUNG, J. C., LIM, E., KIM, S. H., KIM, S., JUNG, M., \& OH, S Pratical synthes is and biological evaluation of bergenin analogs. ChemBiol, Drug Des, 2011, 78:725-729.

4. LIM, H., KIM, H., KIM, S., CHANG, M., RHEE, G. P., CHOI, J. Protective effects of acetylbergenin against carbon tetrachlorideinduced hepatotoxicity in rats. Pharmacology, Toxicology \&Pharmaceutic, 24(2): 114-118, 2001.

5. LIM, S., KIM, H., CHOI, H., OH, S., JANG, C. G., CHOI, W. $\mathrm{J}$. Effects of acetylbergenin against D- galactosamine induced hepatotoxicity in rats. Pharmacological Research , 42 (5): 471474, 2000.

6. NAZIR, N., KOUL, S., QURISHI, M. A., NAJAR, M. H., ZARGAR, M. I. . Evaluation of antioxidant and antimicrobial activities of Bergenin and is derivatives obtained by chemoenzymatic synthesis. European Journal Medicinal Chemistry, V. 46, p.2415-2420. 2011.

7. QUIN, X., YANG, Y., FAN, T., GONG, T., ZHANG, X., \& HUANG, Y. Preparation, characterization and in vivo evaluation of bergenin phospholipid complex.Acta Pharmacologica Sinica,31:127-136, 2010.

8. SANTOS, D. R. Biotrasformation of bergenin using Amazon fungi as biocatalyst. Masters dissertation UEA, 2012.

9. FRICK, W., HOFMANN J., FISCHER, H., SCHMIDT, R. R. The structure of bergenin, Carbohydrate Research, 210:71-77, 1991.

10. OBIRKS, J. B.; Photophysics of Aromatic Molecules, John Wiley \& Sons Ltd., New York, 1970.

11. FUKUI, K. T., YONEZAWA, T., SHINGU, H. . A Molecular Orbital Theory of Reactivity in Aromatic Hydrocarbons.J. Chem. Phys, p.722-725. 1952

\section{Gisele Franco de Castro ${ }^{*}$, Diego dos Santos Freitas ${ }^{a}$,Alberto dos Santos Marques ${ }^{\text {b }}$ \& YujiTakahata.}

anniversidade do Estado do Amazonas, Centro de Estudos Superiores de Tefe, AM.

bUniversidade Federal do Amazonas, Laboratório de Tecnologia com Moléculas Bioativas, LTMB, Departamento de Química.

*E-mail: francogisele@gmail.com 